\title{
PDGFR $\alpha$ Expression Distinguishes GFAP-Expressing Neural Stem Cells from PDGF-Responsive Neural Precursors in the Adult Periventricular Area
}

\author{
Andrew Chojnacki, Gloria Mak, and Samuel Weiss \\ Hotchkiss Brain Institute and Department of Cell Biology and Anatomy, Faculty of Medicine, University of Calgary, Calgary, Alberta T2N 4N1, Canada
}

Jackson et al. (2006) have reported that adult glial fibrillary acid protein (GFAP)-expressing neural stem cells (NSCs) also express platelet-derived growth factor (PDGF) receptor- $\alpha$ (PDGFR $\alpha$ ), and that their stimulation by PDGF induced the formation of a glioma-like mass. Here, we reexamined the relationship between PDGFR $\alpha$ and GFAP expression within the three-dimensional organization of the adult periventricular area. Using four independent PDGFR $\alpha$ antibodies, we found that adult mouse GFAP-expressing NSCs and PDGFR $\alpha$-expressing cells represent two distinct populations of neural precursors. Examination of the adult periventricular area in a mouse line that expresses nuclear-localized enhanced green fluorescent protein under the control of the PDGFR $\alpha$ promoter confirmed that GFAP-expressing NSCs do not express PDGFR $\alpha$. Furthermore, PDGF-responsive neural precursors were found at least one cell layer subjacent to the ependymal layer, and were evenly distributed across the lateral ventricular wall, which contrasts with the reported patchy and often ependymal localization of adult GFAP-expressing NSCs. Adult human PDGFR $\alpha$-expressing neural precursors were also found not to express GFAP. PDGF-responsive neural precursors, but not GFAP-expressing NSCs, responded to infusions of PDGF by generating glioma-like masses. Our results do not support the view that GFAP-expressing NSCs are the origin of glioma-like masses that form after intraventricular PDGF infusion.

\section{Introduction}

Adult neural stem cells (NSCs) are targets of strategies for CNS repair after injury or disease and may contribute to brain tumor formation. NSCs in the adult rodent brain reside in the periventricular area of the lateral ventricles (Reynolds and Weiss, 1992) and the hippocampus (Palmer et al., 1997). Immunological and cytoarchitectural analyses have revealed that adult periventricular NSCs express GFAP, are located within the ependymal and subependymal layers, and are concentrated in pockets along the medial and lateral walls of the lateral ventricles (Doetsch et al., 1999; Merkle et al., 2007; Mirzadeh et al., 2008; Shen et al., 2008). NSCs also populate the adult human hippocampus (Eriksson et al., 1998; Kukekov et al., 1999; Arsenijevic et al., 2001) and periventricular area (Kirschenbaum et al., 1994; Pincus et al., 1997; Sanai et al., 2004; Curtis et al., 2007).

Several distinct populations of neural precursors that express platelet-derived growth factor receptor- $\alpha$ (PDGFR $\alpha$ ) reside throughout the mouse and human brain (Rakic and Zecevic,

\footnotetext{
Received March 25, 2011; revised May 10, 2011; accepted May 13, 2011.

Author contributions: A.C. and G.M. designed research; A.C. and G.M. performed research; A.C. analyzed data; A.C. and S.W. wrote the paper.

This work was supported by the Canadian Institutes of Health Research and studentship (G.M.) and scientist (S.W.) awards from the Alberta Heritage Foundation for Medical Research. We thank M. Gotz and D. van der Kooy for comments on earlier versions of this manuscript.

G. Mak's present address: Department of Medical Genetics, Centre for Molecular Medicine and Therapeutics, University of British Colombia, Room 2034, 950 West 28th Avenue, Vancouver, BC V5Z 4H4, Canada.

Correspondence should be addressed to Samuel Weiss, 1 A10 Health Research Innovation Centre, 3330 Hospital Drive NW, Calgary, Alberta T2N 4N1, Canada. E-mail: weiss@ucalgary.ca.

DOI:10.1523/JNEUROSCI.1531-11.2011

Copyright $\odot 2011$ the authors $\quad 0270-6474 / 11 / 319503-10 \$ 15.00 / 0$
}

2003; Kessaris et al., 2006; Parras et al., 2007). Termed oligodendrocyte progenitor cells (OPCs) and PDGF-responsive neural precursors (PRPs) (Chojnacki and Weiss, 2004), they are slowly dividing and primarily generate oligodendrocytes (ffrenchConstant and Raff, 1986; Wolswijk and Noble, 1989; Gregg et al., 2007). OPCs/PRPs and adult periventricular NSCs are also thought to be two distinct neural precursor populations.

Expression of PDGFR $\alpha$ and the lack of GFAP expression by OPCs/PRPs used to distinguish them from GFAP-expressing periventricular NSCs. Adult periventricular NSCs were initially isolated by their dependence on epidermal growth factor (EGF) to proliferate and form spherical clusters of undifferentiated cells, termed neurospheres (Reynolds and Weiss, 1992). It was later shown that GFAP-expressing NSCs activated by the cytosine arabinoside-induced death of their progeny, the transit-amplifying progenitors, express the EGF receptor (EGFR) (Pastrana et al., 2009). Furthermore, EGFR and PDGFR $\alpha$ label distinct neural precursor populations during embryonic development (Chojnacki and Weiss, 2004) and in the adult periventricular area (Jackson et al., 2006). However, postnatal and adult OPCs/PRPs have now been reported to express EGFR (Aguirre et al., 2004). Similarly, NG2 expression, previously described as specific for OPCs/ PRPs (Nishiyama et al., 1996), has been found in EGFR-expressing transit-amplifying progenitors (Aguirre et al., 2004), although this has been disputed (Cesetti et al., 2009; Komitova et al., 2009). Recently, PDGFR $\alpha$ has been reported to be expressed by a subset of adult periventricular GFAP-expressing NSCs (Jackson et al., 2006), suggesting that it is not specific to OPCs/PRPs. This observation and the formation of glioma-like masses after intraventricular infusions 


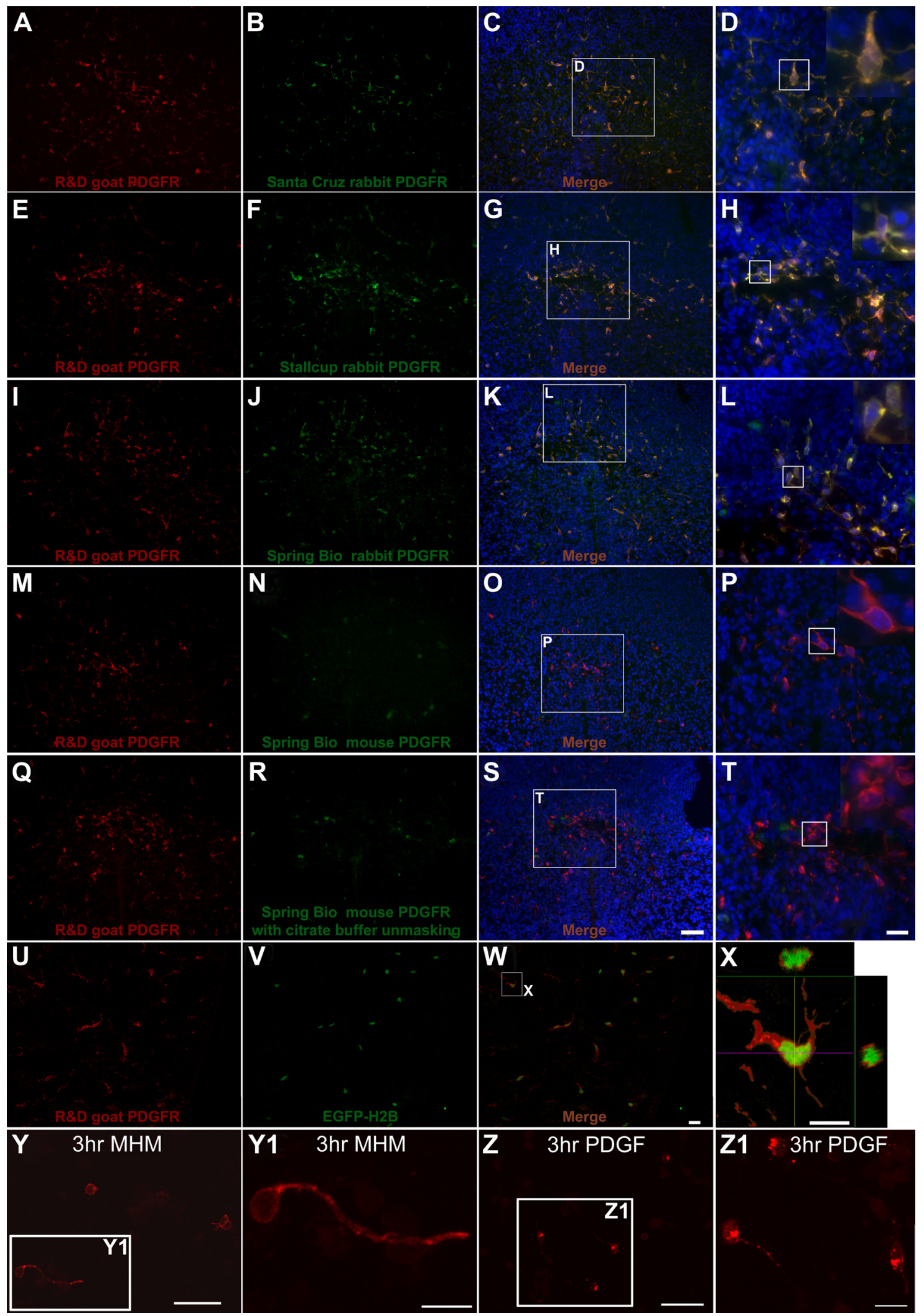

Figure 1. Identification of PDGFR $\alpha$ expression in the E14 ventral forebrain using multiple antibodies directed against the protein. PDGFR $\alpha$ expression was examined in cryosections of the E14 ventral forebrain by immunohistochemistry using commercially available antibodies obtained from R\&D Systems $(\boldsymbol{A}, \boldsymbol{E}, \boldsymbol{I}, \boldsymbol{M}, \boldsymbol{Q}, \boldsymbol{U}$ and $\boldsymbol{C}, \boldsymbol{D}, \boldsymbol{G}, \boldsymbol{H}, \boldsymbol{K}, \boldsymbol{L}, \boldsymbol{O}, \boldsymbol{P}, \boldsymbol{S}, \boldsymbol{I}, \boldsymbol{W}, \boldsymbol{X})$, Santa $\mathrm{Cruz}(\boldsymbol{B}-\boldsymbol{D})$, and Spring Bioscience $(\boldsymbol{J}-\boldsymbol{L}$ for rabbit-derived antibody and $\boldsymbol{N}-\boldsymbol{P}$ and $\boldsymbol{R}$ - $\boldsymbol{T}$ for mouse-derived antibody) as well as a rabbit anti-PDGFR $\alpha$ antibody obtained from Dr. Bill Stallcup $(\boldsymbol{F}-\boldsymbol{H})$. Notethat the R\&D Systems anti-mousePDGFR $\alpha$ antibody colabels with all the other PDGFR $\alpha$ antibodies examined except for the Spring Bioscience mouse PDGFR $\alpha$ antibody. The recommended citrate buffer unmasking wash had no impact on the ability of the Spring Bioscience mouse PDGFR $\alpha$ antibody to detect the protein $(\mathbf{Q}-\boldsymbol{T})$. The R\&D Systems anti-mouse PDGFR $\alpha$ antibody labels cells that express an EGFP-H2B fusion protein whose expression is driven by the endogenous PDGFR $\alpha$ promoter $(\boldsymbol{U}-\boldsymbol{X})$. In addition, PDGFR $\alpha$ expression as detected by the R\&D Systems antibody became internalized (compare $\boldsymbol{Y}-\boldsymbol{Y} \mathbf{1}$ to $Z \mathbf{Z}-\mathbf{Z 1}$ ) after 3 h of PDGF treatment in cells from postnatal day zero dissociated brain tissue, further confirming its specificity for PDGFR $\alpha$. Scale bars:S (for $A-C, E-G, I-K, M-0, Q-S), 50 \mu \mathrm{m} ; I($ for $D, H, L, P, T), W($ for $U-W), 20 \mu \mathrm{m} ; \boldsymbol{X}, 10 \mu \mathrm{m} ; Y, Z, 30 \mu \mathrm{m} ; Y 1, Z 1,10 \mu \mathrm{m}$. 


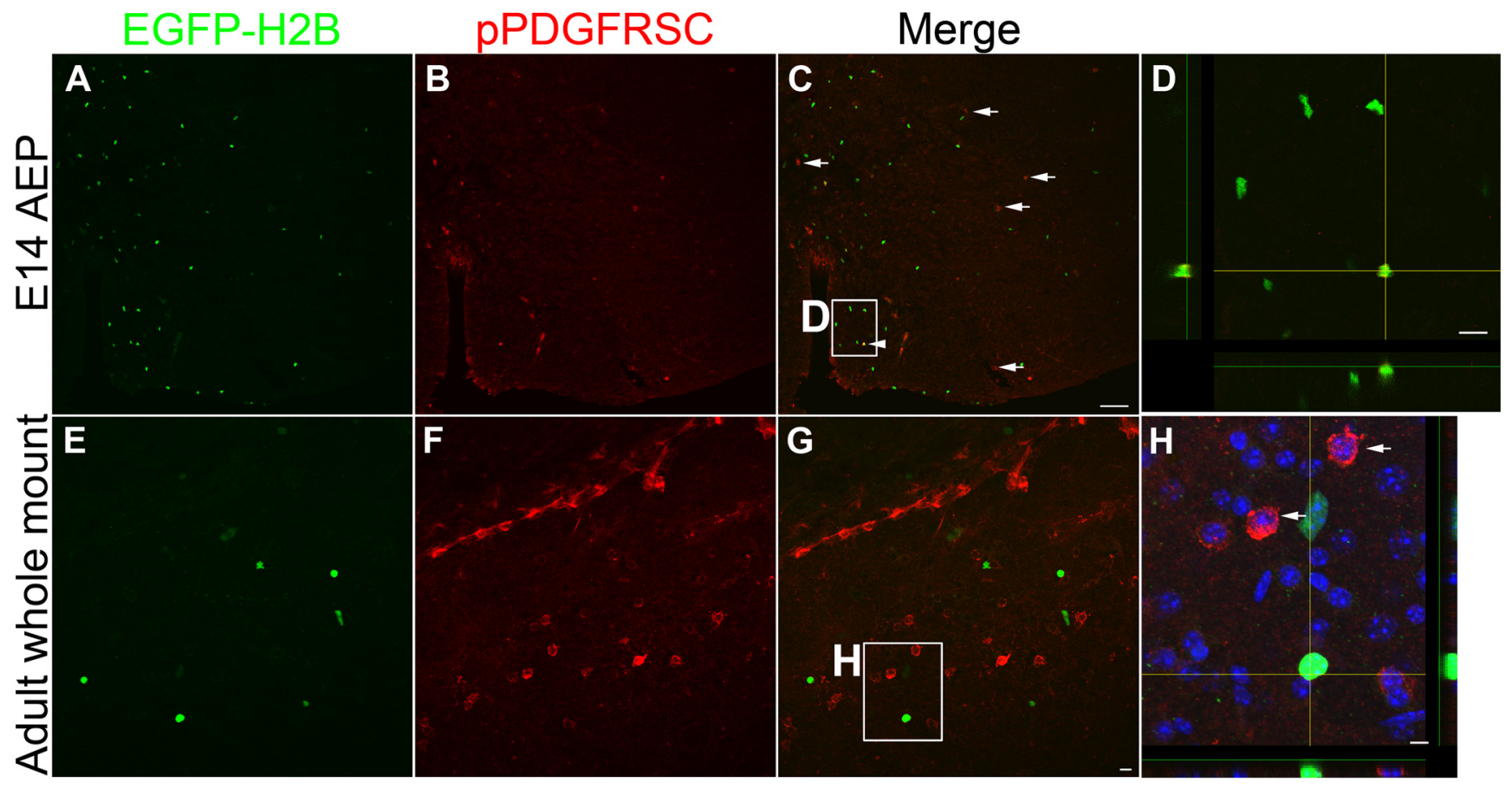

Figure 2. Antibody SC-12910 staining for phospho-PDGFR $\alpha$ does not colocalize with PDGFR $\alpha$-driven EGFP-H2B expression in embryonic day 14 or adult mouse brain. A-D, SC-12910, a phospho-PDGFR $\alpha$-specific antibody (pPDGFRSC, red), failed to colabel cells expressing PDGFR $\alpha$ within the anterior entopeduncular area (AEP) as revealed by their expression of EGFP-H2B (green). A few cells colabeled for EGFP-H2B and SC-12910 (arrowhead in ( and D). In contrast, the majority of the cells labeled by SC-12910 within the E14 brain lacked any EGFP-H2B expression (C, arrows), indicating that the antibody does not specifically detect phospho-PDGFR $\alpha$. E-H, Similarly, SC-12910 labeled cells within whole mounts of the adult periventricular area that did not expres EGFP-H2B ( $\boldsymbol{H}$, arrows), again indicating that it does not specifically detect phospho-PDGFR $\alpha$ in the adult mouse brain. Scale bars: $\boldsymbol{A}-\boldsymbol{C}$ (in $\boldsymbol{C}$ ), $50 \mu \mathrm{m} ; \boldsymbol{D}, 10 \mu \mathrm{m} ; \boldsymbol{E}-\boldsymbol{G}$ (in $\boldsymbol{G}$ ), $10 \mu \mathrm{m} ; \boldsymbol{H}, 5 \mu \mathrm{m}$.

of PDGF led Jackson et al. (2006) to conclude that adult periventricular GFAP-expressing NSCs are a potential source for $\operatorname{PDGFR} \alpha$-overexpressing brain tumors. However, it has now been reported that the adult ventricular surface contains multiple distinct populations of NSCs (Merkle et al., 2007), but whether there are differences in PDGFR $\alpha$ expression between these populations has not been examined.

To determine whether there are differences in PDGFR $\alpha$ expression among the multiple adult periventricular NSC populations, we reexamined the expression of PDGFR $\alpha$ in the adult periventricular area. Using both immune- and transgenic mousebased techniques, we found that neither adult mouse nor human GFAP-expressing NSCs expressed PDGFR $\alpha$. Furthermore, PDGFR $\alpha$ expression was found to be evenly distributed along the ventricular wall, and at least one cell layer subjacent to the ependymal layer, in contrast to the reported distribution of GFAP-expressing NSCs (Mirzadeh et al., 2008; Shen et al., 2008). In addition, only parenchymal infusion of PDGF generated glioma-like masses, whereas intraventricular infusions had no noticeable effect on proliferation. Our results suggest that PDGFR $\alpha$ expression distinguishes between OPCs/PRPs and adult GFAPexpressing NSCs.

\section{Materials and Methods}

Animals. CD-1 mice stocks were maintained in the University of Calgary Animal Resources Center. B6.129S4-Pdgfra ${ }^{\text {tm11(EGFP)Sor } / J ~ m i c e ~ w e r e ~ o b-~}$ tained from The Jackson Laboratory and stocks were maintained in the University of Calgary Animal Resources Center.

Human brain tissue. Human fetal [21, 21, and 17 gestational weeks (g.w.)] brain tissue was obtained from therapeutic abortions. Adult brain tissue [corpus callosum (CC) from a 20 year old and a 16 year old, temporal lobe periventricular area from a 35 year old] was obtained at the time of resection from the Foothills Hospital, Calgary, Alberta.
Immunohistochemistry. Embryonic day 14 (E14) mouse brains were fixed for $4 \mathrm{~h}$ with ice-cold $4 \%$ paraformaldehyde. Twelve-micrometer sections were obtained after the fixed brains were incubated for $24 \mathrm{~h}$ in $10 \%$ sucrose followed by $24 \mathrm{~h}$ in $25 \%$ sucrose, and mounted in OCT (Tissue Tek). Adult male CD1 mice were killed by an intraperitoneal injection of sodium pentobarbital $(11 \mathrm{mg})$ followed by transcardial perfusion with $20 \mathrm{ml}$ of ice-cold PBS, $20 \mathrm{ml}$ of ice-cold $4 \%$ paraformaldehyde, and an $8 \mathrm{~h}$ postfix in ice-cold $4 \%$ paraformaldehyde. For periventricular area staining, sections $(30 \mu \mathrm{m})$ were obtained using a Leica VT100S vibratome. Human fetal and adult brain tissue was fixed for $24 \mathrm{~h}$ in ice-cold $4 \%$ paraformaldehyde and processed for sectioning as described for E14 mouse brains. Some sections processed for staining with the Spring Bioscience antibodies received a 10 min boiling citrate buffer wash. E14 mouse brain sections were then incubated with the primary antibodies for $2 \mathrm{~h}$ at $37^{\circ} \mathrm{C}$ diluted in PBS containing $0.3 \%$ Triton X-100 (if diluted) at the following dilutions: R\&D Systems goat antimouse PDGFR $\alpha$ (1:10), Santa Cruz rabbit anti-PDGFR $\alpha$ (1:10), Spring Bioscience mouse-anti PDGFR $\alpha$ (neat), Spring Bioscience rabbit antiPDGFR $\alpha$ (neat; lot \#s 61212 and 70629), Stallcup rabbit anti-PDGFR $\alpha$ (1:100; kind gift from Dr. Bill Stallcup, Sanford-Burnham Medical Research Institute, La Jolla, CA), and Santa Cruz goat anti-phospho PDGFR $\alpha$ (SC-12910: 1:50). Sections were then incubated for $1 \mathrm{~h}$ at room temperature in PBS containing 10\% normal donkey serum (NDS) followed by a $1 \mathrm{~h}$ incubation at $37^{\circ} \mathrm{C}$ with the appropriate donkey secondary antibodies (all from Jackson ImmunoResearch). Adult mouse brain floating vibratome sections were incubated for $24 \mathrm{~h}$ at room temperature with the primary antibodies in PBS containing $0.3 \%$ Triton X-100 (PBST: if diluted) at the following dilutions: Millipore Bioscience Research Reagents (MBRR) mouse anti-GFAP (1:1000), BTI rabbit antiGFAP (1:400), R\&D Systems goat anti-mouse PDGFR $\alpha$ (1:50), Santa Cruz rabbit anti-PDGFR $\alpha$ (1:100), Spring Bioscience mouse-anti PDGFR $\alpha$ (neat), Spring Bioscience rabbit anti-PDGFR $\alpha$ (1:10; lot \# 61212), and Stallcup rabbit anti-PDGFR $\alpha$ (1:200). Sections were then incubated for $1 \mathrm{~h}$ at room temperature in PBS containing 10\% NDS followed by a $1 \mathrm{~h}$ incubation at $37^{\circ} \mathrm{C}$ with the appropriate donkey sec- 

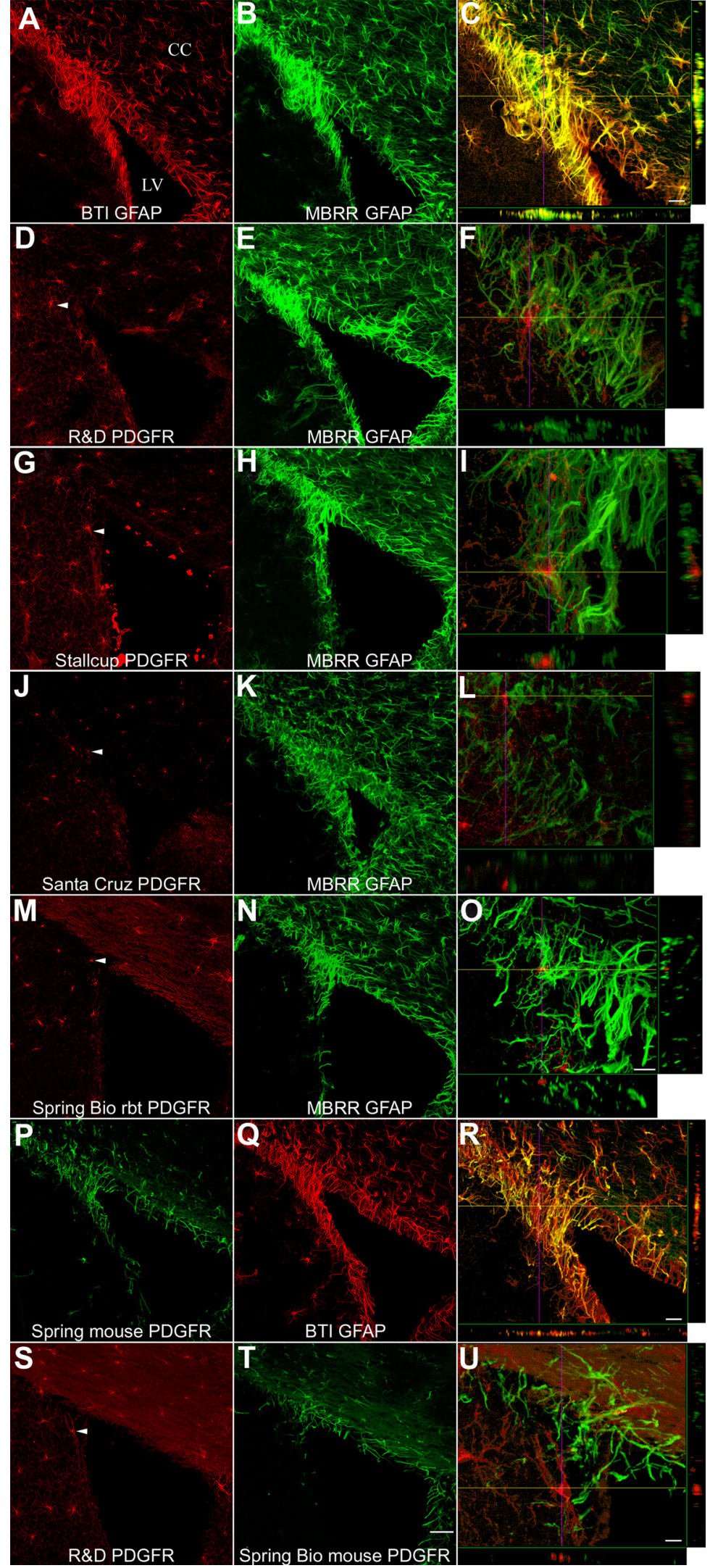

Figure 3. Periventricular area astrocytes do not express PDGFR $\alpha$. Expression of GFAP $(\boldsymbol{A}-\boldsymbol{C}, \boldsymbol{E}, \boldsymbol{H}, \boldsymbol{K}, \boldsymbol{N}, \mathbf{Q})$ was examined by immunohistochemistry in coronal vibratome sections of the adult periventricular area. Confocal microscopy revealed that both the BTI rabbit anti-GFAP $(\boldsymbol{A})$ and the MBRR mouse anti-GFAP $(\boldsymbol{B})$ antibodies detected the same protein, which resulted in clear colabeling in the periventricular area and corpus callosum (C). Cells coexpressing PDGFR $\alpha$ and GFAP were not detected when using the R\&D Systems $(\boldsymbol{D}-\boldsymbol{F})$, Stallcup $(\boldsymbol{G}-\boldsymbol{I})$, Santa Cruz $(\boldsymbol{J}-\boldsymbol{L})$, or the Spring Bioscience rabbit $(\boldsymbol{M}-\mathbf{0})$ PDGFR $\alpha$ antibodies. The Spring ondary antibodies. For the infusion experiments, once fixed the brains were incubated for $24 \mathrm{~h}$ in $10 \%$ sucrose followed by $24 \mathrm{~h}$ in $25 \%$ sucrose, and mounted in OCT (Tissue Tek). Staining of the sections followed the same procedure used for the embryonic mouse brain sections using the primary antibodies at the following dilutions: R\&D Systems goat antimouse PDGFR $\alpha$ (1:10), MBRR mouse antiGFAP (1:1000), and MBRR rabbit anti-NG2 (1:200). For BrdU (Serotec rat anti-BrdU; 1:100) and PDGFR $\alpha$ (R\&D System; 1:10), double-labeling sections were incubated in acetone for $30 \mathrm{~s}$ at room temperature, $20 \mathrm{~min}$ in $1 \mathrm{~N} \mathrm{HCl}$ at $60^{\circ} \mathrm{C}, 24 \mathrm{~h}$ incubation at room temperature with primary antibodies, $1 \mathrm{~h}$ in PBS containing $10 \%$ NDS, and $2 \mathrm{~h}$ at room temperature in the appropriate secondary antibodies. This was followed by staining with the appropriate secondary antibodies for $24 \mathrm{~h}$ at $4^{\circ} \mathrm{C}$ in PBS containing $0.3 \%$ Triton $\mathrm{X}-100$ as well as Hoechst 33258 added for $1 \mathrm{~h}$ for the first wash. Staining of human brain tissue followed the same procedure used for E14 mouse brain sections except using the primary antibodies at the following dilutions: MBRR mouse anti-GFAP (1:500), R\&D Systems goat anti-human PDGFR $\alpha$ (1:20), Spring Bioscience rabbit anti$\operatorname{PDGFR} \alpha$ (neat). Fluorescence-labeled slides were mounted with Fluorsave (Calbiochem). Slides processed for hematoxylin and eosin staining were mounted with Cytoseal XYL (Richard-Allan Scientific). Postnatal day 0 mouse cortices were dissociated and plated on poly-L-ornithine-coated glass coverslips and cultured in a serum-free media hormone mix (MHM) (Chojnacki and Weiss, 2008). After $24 \mathrm{~h}$, the cells were either left in MHM or stimulated with PDGF-AA ( $100 \mathrm{ng} / \mathrm{ml})$ for $3 \mathrm{~h}$. The cells were then fixed for $20 \mathrm{~min}$ in ice-cold $4 \%$ paraformaldehyde and stained for PDGFR $\alpha$ expression using the R\&D systems goat-antimouse PDGFR $\alpha$ antibody (1:20). Images were captured on an Axiocam camera (Zeiss) mounted on a Zeiss Axioplan2 microscope (Zeiss) using Axiovision software, and figures were composed in Adobe Photoshop 6.0 (Adobe Systems). Confocal images where captured on an Olympus Optical Fluoview BX-50 laser scanning confocal microscope using Fluoview software.

Whole mounts of the adult lateral ventricular wall from B6.129S4-Pdgfra ${ }^{\text {tm11(EGFP)Sor } / J ~}$ mice were dissected out as described by Mirzadeh et al.(2008) and then fixed for $30 \mathrm{~min}$ in $4 \%$ ice-cold paraformaldehyde as described by Shen et al.(2008). Whole mounts were then rinsed four times for $15 \mathrm{~min}$ per wash with PBS followed by a $1 \mathrm{~h}$ block at room temperature in PBST containing $10 \%$ NDS. This was followed by a $24 \mathrm{~h}$ incubation at $4^{\circ} \mathrm{C}$ in PBST containing

$\longleftarrow$

Bioscience mouse anti-PDGFR $\alpha$ antibody colocalized with GFAP $(\boldsymbol{P}-\boldsymbol{R})$, but failed to colocalize with PDGFR $\alpha$ as detected by the R\&D Systems antibody ( $\boldsymbol{S}-\boldsymbol{U}$ ). Scale bars: (in $\boldsymbol{I}) \boldsymbol{A}, \boldsymbol{B}, \boldsymbol{D}, \boldsymbol{E}, \boldsymbol{G}$, $H, J, K, M, N, P, Q, S, T, 50 \mu \mathrm{m} ; \boldsymbol{C}, 20 \mu \mathrm{m}$; (in O) $\boldsymbol{F}, \mathbf{I}, \mathbf{L}, \mathbf{O}, 20 \mu \mathrm{m}$; $R, 20 \mu \mathrm{m} ; \boldsymbol{U}, 10 \mu \mathrm{m}$. 

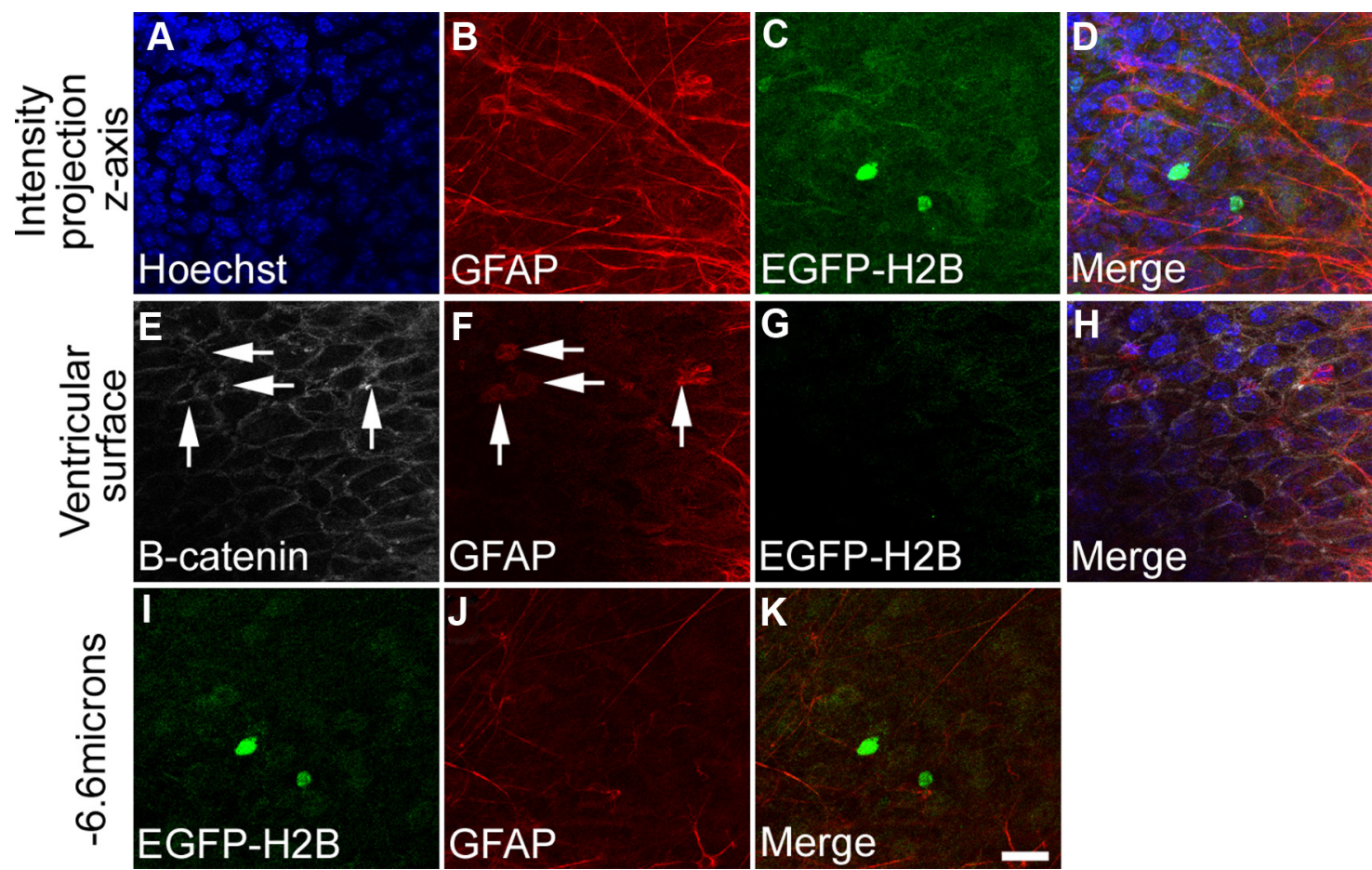

Figure 4. Adult periventricular GFAP-expressing NSCS do not express PDGFR $\alpha$. A flattened $z$-stack $(A-D)$ taken within the posterior dorsal portion of the lateral ventricular wall from a

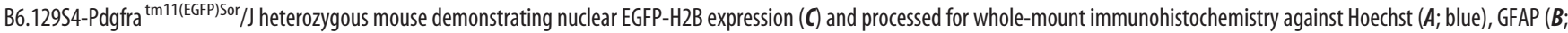
red), and $\beta$-catenin ( $\boldsymbol{E}$; white). GFAP-expressing NSCs identified by their contact with the ventricular surface (arrows in $\boldsymbol{E}$ and $\boldsymbol{F}$ ), did not express EGFP-H2B driven by the endogenous PDGFR $\alpha$ $\operatorname{promoter}(\boldsymbol{D}, \boldsymbol{H}, \boldsymbol{K})$. Scale bar, $20 \mu \mathrm{m}$.

$10 \%$ NDS with rabbit anti-GFAP (BTI; 1:400), mouse anti- $\beta$-catenin (BD Biosciences; 1:500), or Santa Cruz goat anti-phospho PDGFR $\alpha$ (SC12910: 1:50). Afterward, whole mounts were rinsed four times with PBST (15 min per wash) and then incubated for $2 \mathrm{~h}$ at room temperature in the appropriate secondary antibodies. This was followed by four $15 \mathrm{~min}$ washes in PBST and a $1 \mathrm{~h}$ incubation with Hoechst 33258. Whole mounts were mounted in Fluorsave. Six-hundred-nanometer confocal sections of whole mounts were captured on an Olympus Optical Fluoview 1000 laser scanning confocal microscope using Fluoview software.

PDGF infusions and BrdU administration. Male CD1 mice (8-9 weeks) were anesthetized with a 1:10 dilution of sodium pentobarbital in saline (120 mg per kg of body weight, i.p.) and implanted with an osmotic pump (Alzet 1007D, Alza Corp.), placed dorsal subcutaneously. PDGF-AA (human recombinant; R\&D Systems and Peprotech) was dissolved in $0.9 \%$ saline containing $1 \mathrm{mg} / \mathrm{ml}$ bovine serum albumin (Sigma) at a concentration of $3.3 \mathrm{ng} / \mu \mathrm{l}$. Mice received an infusion of $40 \mathrm{ng}$ of PDGF-AA per day, as the manufacturer-specified flow rate is $0.5 \mu \mathrm{l} / \mathrm{h}$. Control mice were implanted with an osmotic pump containing $1 \mathrm{mg} / \mathrm{ml}$ bovine serum albumin (Sigma). The contents of the osmotic pump were channeled though tubing attached to a cannula that was implanted into either the lateral ventricles of the brain $(+0.2 \mathrm{~mm}$ anterior/posterior, $+0.8 \mathrm{~mm}$ medial/lateral lateral, and dorsoventral $-2.5 \mathrm{~mm}$ below the dura with the skull leveled between lambda and bregma) or the parenchyma lateral to the lateral ventricle $(+0.2 \mathrm{~mm}$ anterior/posterior, +2.0 $\mathrm{mm}$ medial/lateral lateral, and dorsoventral $-2.5 \mathrm{~mm}$ below the dura with the skull leveled between lambda and bregma). Each animal was infused for 7 consecutive days. A minimum of three mice were infused per group. One hour before the animals were killed, 1 injection of BrdU (Sigma, $120 \mathrm{mg}$ per kg, dissolved in $0.007 \% \mathrm{NaOH}$ in phosphate buffer) was administered intraperitoneally.

\section{Results}

Identification of bona fide PDGFR $\alpha$ expression in the embryonic mouse forebrain

To determine whether PDGFR $\alpha$ expression can be used to distinguish between OPCs/PRPs and adult periventricular GFAP- expressing NSCs, we tested the specificity of five different antibodies directed against the protein. We tested an $R \& D$ Systems goat anti-mouse PDGFR $\alpha$ and a Santa Cruz rabbit anti-PDGFR $\alpha$ antibody (Chojnacki and Weiss, 2004), an antiPDGFR $\alpha$ from Dr. Bill Stallcup (rabbit; ectodomain) (Nishiyama et al., 1996), and two Spring Biosciences antibodies (mouse and rabbit derived) used by Jackson et al. (2006). With the antibodies, we stained frozen sections of the E14 ventral forebrain wherein PDGFR $\alpha$ mRNA has been demonstrated to be present (Pringle and Richardson, 1993). Both the R\&D Systems (Fig. $1 A$, red) and Santa Cruz (Fig. $1 B$, green) antibodies detected cells within the E14 ventral forebrain, and dual labeling revealed that they labeled the same population of cells (Fig. 1C,D, orange). This suggested that true PDGFR $\alpha$ expression was being detected by the R\&D Systems and Santa Cruz antibodies. Both the Stallcup (Fig. 1F) and Spring Biosciences rabbit (Fig. $1 \mathrm{~J}$ ) antibodies colabeled with the R\&D Systems PDGFR $\alpha$ antibody (Fig. $1 E-L$ ), suggesting that these antibodies also detected bona fide PDGFR $\alpha$ expression. In contrast, the Spring Bioscience mouse-anti PDGFR $\alpha$ antibody failed to strongly label cells in the ventral forebrain (Fig. $1 M-P$ ), regardless of whether the tissue was unmasked using a manufacturerrecommended citrate buffer wash (Fig. 1Q-T). The faint staining that was observed did not colabel with R\&D Systems' goat antimouse PDGFR $\alpha$ antibody (Fig. 1Q-T).

We further confirmed that the R\&D Systems goat anti-mouse PDGFR $\alpha$ antibody was specifically detecting PDGFR $\alpha$ expres-

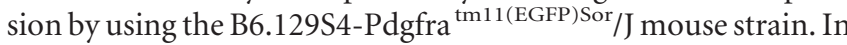
this strain of mice, the endogenous PDGFR $\alpha$ promoter drives the expression of an EGFP-H2B fusion protein, which targets EGFP$\mathrm{H} 2 \mathrm{~B}$ to the nucleus of PDGFR $\alpha$-expressing cells. In E14 brain sections of B6.129S4-Pdgfra ${ }^{\text {tm11(EGFP)Sor } / J ~ h e t e r o z y g o u s ~ m i c e, ~}$ confocal microscopy revealed that all cells that were labeled by the R\&D Systems goat anti-mouse PDGFR $\alpha$ antibody also ex- 
pressed EGFP-H2B (Fig. $1 U-X$ ). In addition, when postnatal day zero mouse cortex was dissociated, plated, and after $24 \mathrm{~h}$ cultured for a further $3 \mathrm{~h}$ in either MHM or PDGF (100 ng/ml), the localization of PDGFR $\alpha$ as detected by the R\&D Systems antibody changed. In the absence of PDGF, PDGFR $\alpha$ staining was membrane bound (Fig. 1Y,Y1), whereas after $3 \mathrm{~h}$ in the presence of PDGF, PDGFR $\alpha$ expression was internalized (Fig. 1Z,Z1). This agrees well with the known internalization of PDGF receptors after ligand binding (Heldin et al., 1982; Coats et al., 1994), and provides further evidence that the R\&D Systems anti-mouse PDGFR $\alpha$ antibody specifically detects the protein. The data suggest that four of the tested antibodies were suitable for determining whether expression of PDGFR $\alpha$ could distinguish between adult periventricular GFAP-expressing NSCs and OPCs/PRPs.

In addition, we examined the specificity of SC-12910, an antibody directed against phosphorylated $\operatorname{PDGFR} \alpha$ that was also used to demonstrate that GFAPexpressing periventricular NSCs express the PDGFR $\alpha$ receptor (Jackson et al., 2006). In E14 brain sections of B6.129S4Pdgfra ${ }^{\text {tml1(EGFP)Sor } / J ~ h e t e r o z y g o u s ~ m i c e, ~}$ confocal microscopy revealed that the majority of EGFP-H2B-expressing cells were not labeled by SC-12910 (Fig. 2A--D). In addition, most of the cells labeled by SC12910 were not EGFP-H2B positive (Fig. $2 C$, arrows), demonstrating the nonspecificity of the antibody. SC-12910 also nonspecifically labeled cells in whole mounts of the adult mouse periventricular area (Fig. $2 E--G$, and arrows in $H$ ). Together, the data suggest that SC-12910 is not suitable for the detection of phosphorylated PDGFR $\alpha$ in immunohistochemistry applications.

\section{PDGFR $\alpha$ is not expressed by adult mouse periventricular GFAP-expressing NSCs}

We reexamined the expression of PDGFR $\alpha$ in the adult mouse brain periventricular area. We first tested two different GFAP antibodies to ensure that we could detect bona fide GFAP expression. Confocal microscopy of sections of the adult mouse periventricular area stained with the MBRR mouse anti-GFAP and BTI rabbit-anti-GFAP antibodies revealed identical staining patterns (Fig. $3 A-C$ ). This suggested that the two antibodies were indeed specific for GFAP. Next, we examined whether any population of cells colabeled for GFAP and PDGFR $\alpha$ expression in 30 $\mu \mathrm{m}$ coronal vibratome sections of the adult mouse periventricular area. All of the antibodies that detected PDGFR $\alpha$ expression in the E14 ventral forebrain, labeled cells that had multiple processes in the corpus callosum and within the periventricular area, reminiscent of OPCs/PRPs (Fig. 3D, G, J, M). Both the R\&D Systems and Stallcup antibodies strongly labeled these cells, whereas the Santa Cruz and Spring Bioscience rabbit antibody showed weaker staining. None of the PDGFR $\alpha$ antibodies colabeled
GFAP-expressing cells (Fig. 3D-O). In contrast, the Spring Bioscience mouse anti-PDGFR $\alpha$ antibody labeled GFAP-expressing periventricular astrocytes (Fig. $3 P-R$ ). Use of the recommended citrate buffer unmasking wash resulted in no detectable staining (data not shown). Furthermore, the Spring Bioscience mouse anti-PDGFR $\alpha$ antibody failed to costain cells labeled with the R\&D Systems anti-mouse PDGFR $\alpha$ antibody in sections of the adult mouse brain (Fig. $3 S-U$ )

Our observations revealed that adult periventricular GFAPexpressing NSCs within the lateral ventricular wall never colabeled for PDGFR $\alpha$ in $30 \mu \mathrm{m}$ coronal sections of the adult mouse brain. However, several different adult periventricular NSCs populations are localized in concentrated pockets along the ventricular wall (Merkle et al., 2007). Therefore, it remained a possibility that the coronal sections we examined missed a subpopulation of GFAP-expressing cells that express PDGFR $\alpha$. In addition, the dense network of GFAP-positive fibers intermixed with PDGFR $\alpha$-positive processes also made it difficult to ensure that we had not missed a minor population of cells expressing both proteins. Therefore, we processed whole mounts of the 


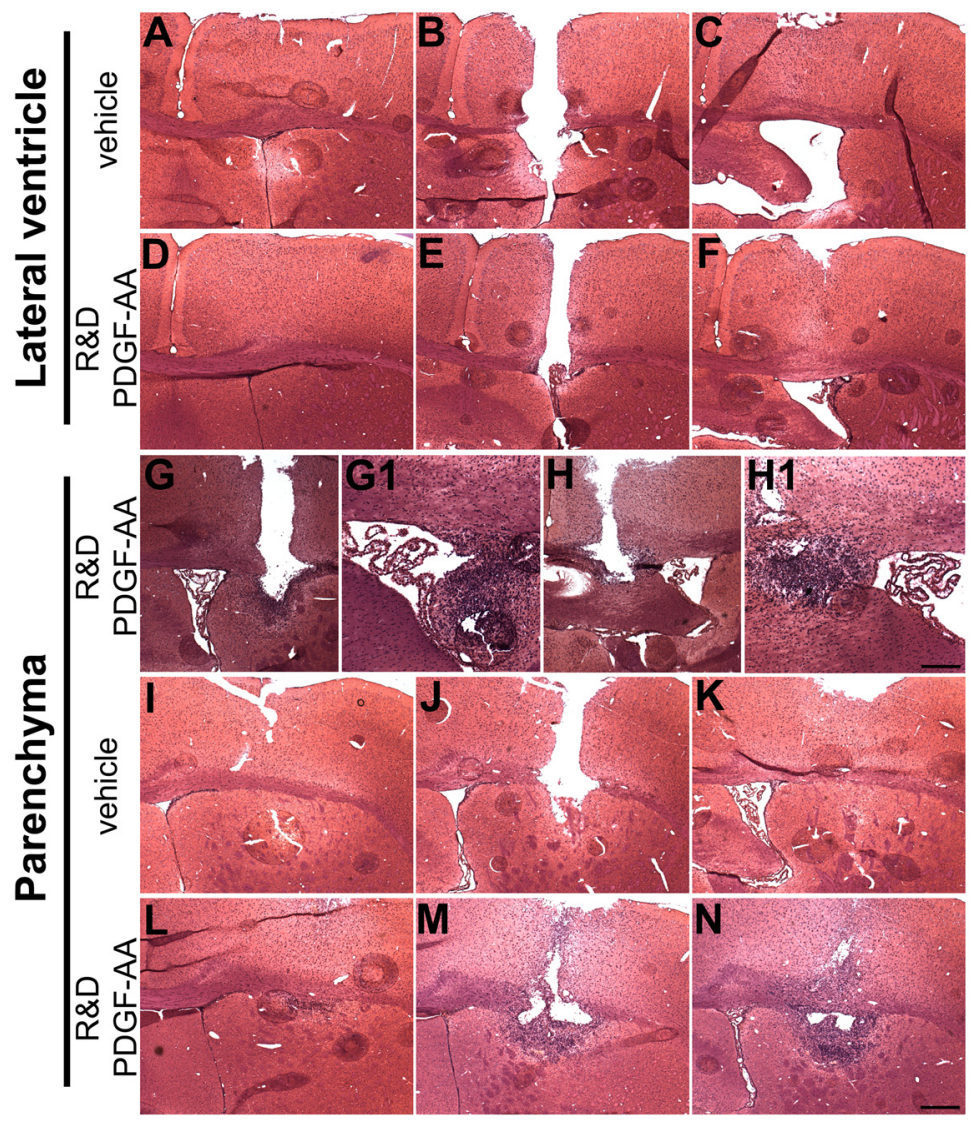

Figure 6. Parenchymal, but not ventricular, infusion of PDGF into the adult mouse brain results in the formation of a cellular mass. Cryosections of adult mouse brains into which vehicle $(\boldsymbol{A}-\boldsymbol{C}, n=3 ; \boldsymbol{I}-\boldsymbol{K}, n=3)$ or PDGF $(\boldsymbol{D}-\boldsymbol{F}, n=7 ; \boldsymbol{L}-\boldsymbol{N}, n=8)$ was infused into the lateral ventricles $(\boldsymbol{A}-\boldsymbol{F})$ or the parenchyma $(\boldsymbol{I}-\boldsymbol{N})$ were processed for hematoxylin and eosin staining. The formation of a cellular mass was not observed when PDGF was infused into the lateral ventricles $(\boldsymbol{D}-\boldsymbol{F})$. Infusions of PDGF that missed the lateral ventricle $(\mathbf{G}-\mathbf{H 1})$, when examined more caudally $(\mathbf{G 1}-\mathbf{H 1})$, gave the appearance of a cellular mass that could be misconstrued as originating from the lateral ventricle. When PDGF was infused into the parenchyma lateral to the lateral ventricle, formation of a cellular mass was observed rostral to $(\boldsymbol{L})$, at $(\boldsymbol{M})$, and caudal to $(\boldsymbol{N})$ the infusion site, in contrast to the vehicle

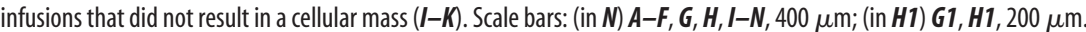

adult lateral ventricular wall obtained from B6.129S4Pdgfra ${ }^{\text {tm11(EGFP)Sor } / J ~ h e t e r o z y g o u s ~ m i c e ~ f o r ~ i m m u n o h i s t o c h e m-~}$ istry against GFAP and $\beta$-catenin (to label cell membranes and identify the ventricular surface). We used these animals specifically because the nuclear localization of EGFP-H2B, indicative of PDGFR $\alpha$ expression, would make it easier to colocalize PDGFR $\alpha$ expression within clearly GFAP-expressing NSCs, should such double-labeled cells exist. Thus, the effect of the dense meshwork of GFAP-positive fibers could be neutralized. Confocal analysis (Fig. 4) revealed that within the posterior dorsal wall of the adult lateral ventricle, an area enriched for GFAP-expressing NSCs (Mirzadeh et al., 2008), no cells expressing EGFP-H2B colabeled for GFAP (three whole-mount ventricular walls from different animals; \#1: 191 EGFP-H2B cells in fifteen $60 \times$ fields; \#2: 100 EGFP-H2B cells in ten $60 \times$ fields; \#3. 71 EGFP-H2B cells in five $60 \times$ fields). Furthermore, lack of PDGFR $\alpha$ and GFAP colabeling was not due to the absence of ventricular surface-contacting GFAP-expressing NSCs within the selected fields. Within whole mounts 2 and 3, we identified 207 GFAP-expressing NSCs (average of 13.8 per field). These cells were either fully intercalated between ependymal cells as described by Shen et al. (2008) or extended a thin process toward the ependymal layer as described by Mirzadeh et al. (2008) (Fig. 4B,E,F). None of the 207 GFAP-expressing NSCs coexpressed PDGFR $\alpha$-driven
EGFP-H2B (Fig. 4D, $H, K$ ). Furthermore, EGFP-H2B-expressing nuclei were always at least one cell layer subjacent to the ependymal layer (Fig. 4I-K). The data suggest that adult GFAP-expressing cells do not express PDGFR $\alpha$, and that PDGFR $\alpha$ expression distinguishes between adult mouse periventricular NSCs and OPCs/PRPs.

PDGFR $\alpha$-expressing cells in the human adult periventricular area do not express GFAP

It is possible that adult periventricular GFAP-expressing NSCs of the adult human brain may have antigen profiles distinct from those of the adult mouse brain. Therefore, we first sought to determine whether we could also detect bona fide PDGFR $\alpha$ expression in sections of the fetal human brain. We used the Spring Bioscience rabbit anti-PDGFR $\alpha$ antibody in conjunction with an R\&D Systems goat anti-human PDGFR $\alpha$ antibody to stain for PDGFR $\alpha$ expression in sections of adult human brain. Confocal microscopy revealed that both antibodies labeled the same population of cells in three different samples of sectioned fetal human brain tissue (Fig. 5A-C). Furthermore, both antibodies labeled cells with multiple processes in two different samples of human corpus callosum (Fig. 5D-F); however, use of the R\&D Systems antibody resulted in stronger and more specific staining. Next, we used the R\&D Systems goat antihuman $\operatorname{PDGFR} \alpha$ antibody together with an anti-GFAP antibody to examine the expression patterns of the proteins in the human temporal lobe periventricular area. Confocal microscopy revealed that PDGFR $\alpha$-expressing cells within the adult human periventricular area did not express GFAP (Fig. 5G-J). Furthermore, we examined the periventricular white matter in the same section, which contained an abundance of PDGFR $\alpha$-expressing cells and cells expressing GFAP, and found that none of the cells expressed both antigens (Fig. $5 K$ ). The data suggest that adult human periventricular GFAPexpressing cells do not express $\operatorname{PDGFR} \alpha$, and that adult human NSCs and OPCs/PRPs can be distinguished from each other by the expression of PDGFR $\alpha$.

\section{Infusion of PDGF-AA into the parenchyma, but not the} lateral ventricle, of the adult mouse brain generates a cellular mass

Jackson et al. (2006) reported that infusion of PDGF into the lateral ventricles led to the generation of a glioma-like mass on either the lateral or medial side of the infused ventricle. This was attributed to the expansion of PDGFR $\alpha$-expressing periventricular GFAP-expressing NSCs. Our examination of PDGFR $\alpha$ and GFAP expression in cells of the adult mouse periventricular area revealed that the two proteins were not coexpressed (Figs. 3, 4). Therefore, it was possible that the generation of a glioma-like mass after intraventricular infusion of PDGF-AA was actually the result of OPC/PRP proliferation. Like Jackson et al. (2006), we 

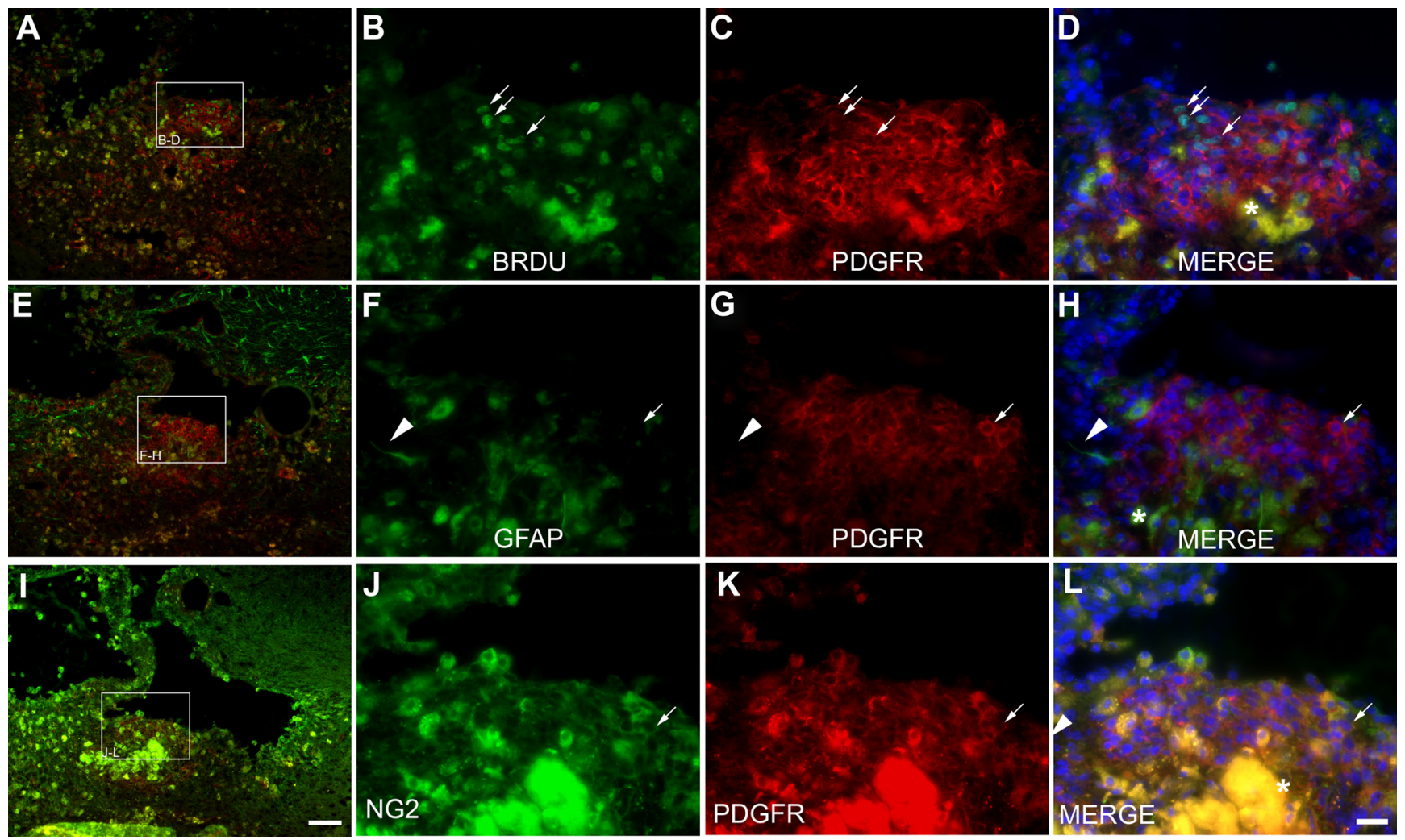

Figure 7. The core of the cellular mass is composed of proliferating PDGFR $\alpha$-expressing neural precursors that lack GFAP expression. Cryosections of an adult mouse brain wherein PDGF was infused parenchymally was stained for PDGFR $\alpha$ (R\&D Systems) and BrdU (A-D), GFAP (MBRR; $\boldsymbol{E}-\boldsymbol{H})$, or NG2 (I-L). Only the core of the cellular mass contained PDGFR $\alpha$-expressing cells that were double labeled for BrdU (arrows in $\boldsymbol{B}-\boldsymbol{D}$ ). These cells were GFAP negative (arrows in $\boldsymbol{F}-\boldsymbol{H}$ ), but labeled for NG2 (arrows in $\boldsymbol{J} \boldsymbol{L}$ ). Some GFAP expression was observed within the core of the mass, but it did not colocalize with PDGFR $\alpha$ expression (arrowheads in $\boldsymbol{F}-\boldsymbol{H}$ ). The asterisks in $\boldsymbol{D}, \boldsymbol{H}$, and $\boldsymbol{L}$ indicate examples of autofluorescent background. Scale bars: (in $\boldsymbol{J}) \boldsymbol{A}, \boldsymbol{E}, \boldsymbol{J}, 50 \mu \mathrm{m} ;$ (in $\boldsymbol{L}) \boldsymbol{B}-\boldsymbol{D}, \boldsymbol{F}-\boldsymbol{H}$, $J-L, 20 \mu \mathrm{m}$.

infused 40 ng of PDGF-AA per day or vehicle control for $7 \mathrm{~d}$ into the lateral ventricles, and examined the infused brains using hematoxylin and eosin staining. We sectioned rostrally and caudally to the infusion site, and found that we had correctly targeted the lateral ventricle as evidenced by the needle tract (Fig. 6A-F). However, we did not observe the formation of a cellular mass on either the lateral or medial side of the ventricle regardless of whether we infused PDGF-AA procured from R\&D Systems (Fig. $6 A-F)(n=3)$ or Peprotech $(n=4$, data not shown). Interestingly, the formation of a cellular mass that could be mistaken as originating from the lateral ventricle was only observed when the cannula missed the lateral ventricle and was infusing PDGF into the parenchyma immediately medial or lateral to it (Fig. 6G-H1). This suggested that OPCs/PRPs, present throughout the parenchyma of the adult brain, were responsible for the generation of cellular masses after PDGF infusion. To test this, we infused PDGF-AA or vehicle control, from either R\&D Systems or Peprotech, into the parenchyma lateral to the lateral ventricle for $7 \mathrm{~d}$, and examined the sections of the infused brains with hematoxylin and eosin staining. Vehicle infusion did not result in the generation of a cellular mass at the infusion site or rostral or caudal to it (Fig. 6I-K). However, PDGF-AA infused into the parenchyma resulted in a cellular mass that extended at least $0.5 \mathrm{~mm}$ rostral and caudal to the site of infusion $(n=8)$ (Fig. $6 L-N)$. The core of the cellular mass was composed of some autofluorescent background (Fig. 7, asterisks) as well as PDGFR $\alpha$-expressing cells that also labeled for BrdU (Fig. $7 A-D$ ), which was administered $1 \mathrm{~h}$ before the mice were killed. Despite the abundant GFAP expression observed in the overlying corpus callosum, these dividing
PDGFR $\alpha$-expressing cells lacked GFAP expression (Fig. 7E-H). However, the PDGFR $\alpha$-expressing cells also labeled for the OPC/ PRP antigen NG2 (Nishiyama et al., 1996) (Fig. 7I-L). Together, the data suggest that the cellular mass generated after PDGF infusion into the adult mouse brain is generated by OPCs/PRPs resident in the parenchyma and not by adult periventricular NSCs.

\section{Discussion}

Jackson et al. (2006) reported that GFAP-expressing NSCs in the adult periventricular area express PDGFR $\alpha$. Furthermore, they found that infusion of PDGF into the mouse lateral ventricles promoted the expansion of the periventricular area into gliomalike masses, which suggested a link between periventricular GFAP-expressing NSCs and gliomas. In contrast, our findings unambiguously demonstrate that neither mouse nor human periventricular area GFAP-expressing NSCs express PDGFR $\alpha$. Furthermore, our results strongly suggest that it is PDGFR $\alpha$ expressing OPCs/PRPs populating the parenchyma of the adult brain that generate cellular masses after PDGF infusions.

In our study several lines of evidence suggest that PDGFR $\alpha$ expression is a feature that distinguishes OPCs/PRPs from adult periventricular GFAP-expressing NSCs. First, examination of GFAP and PDGFR $\alpha$ labeling in the adult periventricular area demonstrated mutually exclusive staining patterns. We were also able to reproduce the double labeling of PDGFR $\alpha$ and GFAP observed by Jackson et al. (2006), but only by using a mouse antibody from Spring Bioscience directed against PDGFR $\alpha$, which we determined does not detect the protein in vivo (Figs. 1, 3). Sec- 
ond, PDGFR $\alpha$ expression, as revealed by EGFP-H2B expression, was evenly distributed along the ventricular wall and was found at least one layer subjacent to ependymal cells. This is in contrast to the reported localized concentrations of adult periventricular GFAP-expressing NSCs along the ventricular surface (Merkle et al., 2007), and the observation that a subpopulation are found intercalated within the ependymal layer (Shen et al., 2008).

Third, presumed expression of PDGFR $\alpha$ by adult periventricular GFAP-expressing NSCs would have predicted their expansion after intraventricular PDGF infusion. In our study, we found that PDGF-AA infused into the lateral ventricle never resulted in the formation of a cellular mass on either the lateral or medial sides of the ventricle. This may in part be because we found that PDGFR $\alpha$-expressing cells were situated at least one cell layer subjacent to the ependymal layer (Fig. 4), and may therefore not have access to signals reaching the ventricular surface. In contrast, parenchymal infusions just lateral or medial to the lateral ventricle gave the appearance of glioma-like masses expanding outward from either the medial or lateral walls of the lateral ventricle. Similarly, the glioma-like masses formed after PDGF infusion originated from either the medial and lateral walls, but not both simultaneously (Jackson et al., 2006). In contrast, intraventricular EGF infusion results in the simultaneous expansion of neural precursors on both the lateral and medial aspects of the ventricle (Craig et al., 1996). Our findings are in agreement with two recently published studies that found that NG2-expressing precursors that presumptively express PDGFR $\alpha$ do not express GFAP in the adult periventricular area (Cesetti et al., 2009; Komitova et al., 2009). However, these studies did not directly address whether a population of GFAP-expressing NSCs expresses PDGFR $\alpha$. Together, these data suggest that the adult OPCs/PRPs, and not adult periventricular NSCs, are the precursors to the formation of cellular masses after PDGF infusion.

Other lines of evidence also support the hypothesis that OPCs/ PRPs and not periventricular GFAP-expressing NSCs generate cellular masses in response to PDGF infusion. Jackson et al. (2006) found that PDGF promoted the generation of primary neurospheres from the adult periventricular area and that it also increased the generation of secondary neurospheres. This led them to conclude that PDGF acted directly on periventricular GFAP-expressing NSCs, to promote their self-renewal. We (Chojnacki and Weiss, 2004) and others (Bögler et al., 1990; McKinnon et al., 1990) have previously reported that FGF can promote the expansion of OPCs/PRPs. Dissections of the adult periventricular area ultimately contain at least two distinct precursor cells: GFAP-expressing NSCs, and OPCs/PRPs. It would be impossible to dissect the subventricular zone free of the OPCs/PRPs that are located throughout the parenchyma and immediately subjacent to the ependymal layer. Therefore, cultures of adult periventricular area in the presence of PDGF-AA and FGF-2 likely stimulate the formation of neurospheres by OPCs/PRPs and GFAP-expressing NSCs, resulting in an increased number of neurospheres generated as compared to FGF2 alone. Furthermore, primary neurospheres passaged into FGF2 and PDGF would also generate more neurospheres, since FGF2-responsive NSCs can also generate PDGFR $\alpha$-expressing neural precursors (Menn et al., 2006) (A. Chojnacki and S. Weiss, unpublished observations). In sum, the results of our study and careful examination of those of Jackson et al. (2006) strongly suggest that only OPCs/PRPs proliferate in vivo in response to PDGF.

Our observations that OPCs/PRPs, but not periventricular GFAP-expressing NSCs, proliferate to generate cellular masses after infusion of PDGF into the brain parenchyma may have implications on the origins of brain tumors. Our work is consistent with that of Assanah et al. (2006), wherein retroviral overexpression of $\mathrm{PDGFB}$, in rat brain white matter, led to the generation of tumors that closely resembled human glioblastomas. They also found that the majority of the cells that populated the tumor expressed OLIG2, NG2, and PDGFR $\alpha$, all markers of OPCs/PRPs. However, $<3 \%$ of the infected cells expressed GFAP. Most glioma cell lines secrete PDGF-A and/or PDGF B, at levels of up to $50 \mathrm{ng} / \mathrm{ml}$ (Nistér et al., 1986; Betsholtz et al., 1989; Assanah et al., 2006), which would be sufficient to drive the proliferation of not only the tumor cells but surrounding genotypically normal OPCs/PRPs in an autocrine/paracrine fashion. Additionally, PDGFR $\alpha$ is overexpressed by human glial tumors (Fleming et al., 1992; Guha et al., 1995). Together with these studies, our results suggest that OPCs/PRPs and not periventricular GFAP-expressing NSCs may be the cell of origin for a subset of tumors wherein an autocrine/paracrine PDGF signaling loop may initially drive their proliferation. We do not exclude the possibility that periventricular GFAPexpressing NSCs or their EGFR-expressing progeny could be cells of origin for other brain tumor subtypes. Nevertheless, our study strongly suggests that introducing mutations identified in secondary glioblastomas into mouse or human PRPs could provide a powerful model for identifying pathways required for their transformation into brain tumor-generating cells.

\section{References}

Aguirre AA, Chittajallu R, Belachew S, Gallo V (2004) NG2-expressing cells in the subventricular zone are type $\mathrm{C}$-like cells and contribute to interneuron generation in the postnatal hippocampus. J Cell Biol 165:575-589.

Arsenijevic Y, Villemure JG, Brunet JF, Bloch JJ, Déglon N, Kostic C, Zurn A, Aebischer P (2001) Isolation of multipotent neural precursors residing in the cortex of the adult human brain. Exp Neurol 170:48-62.

Assanah M, Lochhead R, Ogden A, Bruce J, Goldman J, Canoll P (2006) Glial progenitors in adult white matter are driven to form malignant gliomas by platelet-derived growth factor-expressing retroviruses. J Neurosci 26:6781-6790.

Betsholtz C, Nistér M, Rorsman F, Heldin CH, Westermark B (1989) Structural and functional aspects of platelet-derived growth factor and its role in the pathogenesis of glioblastoma. Mol Chem Neuropathol 10:27-36.

Bögler O, Wren D, Barnett SC, Land H, Noble M (1990) Cooperation between two growth factors promotes extended self-renewal and inhibits differentiation of oligodendrocyte-type- 2 astrocyte (O-2A) progenitor cells. Proc Natl Acad Sci U S A 87:6368-6372.

Cesetti T, Obernier K, Bengtson CP, Fila T, Mandl C, Hölzl-Wenig G, Wörner K, Eckstein V, Ciccolini F (2009) Analysis of stem cell lineage progression in the neonatal subventricular zone identifies EGFR+/NG2- cells as transit-amplifying precursors. Stem Cells 27:1443-1454.

Chojnacki A, Weiss S (2004) Isolation of a novel platelet-derived growth factor-responsive precursor from the embryonic ventral forebrain. J Neurosci 24:10888-10899.

Chojnacki A, Weiss S (2008) Production of neurons, astrocytes and oligodendrocytes from mammalian CNS stem cells. Nat Protoc 3:935-940.

Coats SR, Olashaw NE, Pledger WJ (1994) Characterization of plateletderived growth factor alpha receptor synthesis and metabolic turnover. Cell Growth Differ 5:937-942.

Craig CG, Tropepe V, Morshead CM, Reynolds BA, Weiss S, van der Kooy D (1996) In vivo growth factor expansion of endogenous subependymal neural precursor cell populations in the adult mouse brain. J Neurosci 16:2649-2658.

Curtis MA, Kam M, Nannmark U, Anderson MF, Axell MZ, Wikkelso C, Holtås S, van Roon-Mom WM, Björk-Eriksson T, Nordborg C, Frisén J, Dragunow M, Faull RL, Eriksson PS (2007) Human neuroblasts migrate to the olfactory bulb via a lateral ventricular extension. Science 315:1243-1249

Doetsch F, Caillé I, Lim DA, García-Verdugo JM, Alvarez-Buylla A (1999) Subventricular zone astrocytes are neural stem cells in the adult mammalian brain. Cell 97:703-716. 
Eriksson PS, Perfilieva E, Björk-Eriksson T, Alborn AM, Nordborg C, Peterson DA, Gage FH (1998) Neurogenesis in the adult human hippocampus. Nat Med 4:1313-1317.

ffrench-Constant C, Raff MC (1986) Proliferating bipotential glial progenitor cells in adult rat optic nerve. Nature 319:499-502.

Fleming TP, Saxena A, Clark WC, Robertson JT, Oldfield EH, Aaronson SA, Ali IU (1992) Amplification and/or overexpression of platelet-derived growth factor receptors and epidermal growth factor receptor in human glial tumors. Cancer Res 52:4550-4553.

Gregg C, Shikar V, Larsen P, Mak G, Chojnacki A, Yong VW, Weiss S (2007) White matter plasticity and enhanced remyelination in the maternal CNS. J Neurosci 27:1812-1823.

Guha A, Dashner K, Black PM, Wagner JA, Stiles CD (1995) Expression of PDGF and PDGF receptors in human astrocytoma operation specimens supports the existence of an autocrine loop. Int J Cancer 60:168-173.

Heldin CH, Wasteson A, Westermark B (1982) Interaction of plateletderived growth factor with its fibroblast receptor. Demonstration of ligand degradation and receptor modulation. J Biol Chem 257:4216-4221.

Jackson EL, Garcia-Verdugo JM, Gil-Perotin S, Roy M, Quinones-Hinojosa A, VandenBerg S, Alvarez-Buylla A (2006) PDGFR alpha-positive B cells are neural stem cells in the adult SVZ that form glioma-like growths in response to increased PDGF signaling. Neuron 51:187-199.

Kessaris N, Fogarty M, Iannarelli P, Grist M, Wegner M, Richardson WD (2006) Competing waves of oligodendrocytes in the forebrain and postnatal elimination of an embryonic lineage. Nat Neurosci 9:173-179.

Kirschenbaum B, Nedergaard M, Preuss A, Barami K, Fraser RA, Goldman SA (1994) In vitro neuronal production and differentiation by precursor cells derived from the adult human forebrain. Cereb Cortex $4: 576-589$

Komitova M, Zhu X, Serwanski DR, Nishiyama A (2009) NG2 cells are distinct from neurogenic cells in the postnatal mouse subventricular zone. J Comp Neurol 512:702-716.

Kukekov VG, Laywell ED, Suslov O, Davies K, Scheffler B, Thomas LB, O’Brien TF, Kusakabe M, Steindler DA (1999) Multipotent stem/progenitor cells with similar properties arise from two neurogenic regions of adult human brain. Exp Neurol 156:333-344.

McKinnon RD, Matsui T, Dubois-Dalcq M, Aaronson SA (1990) FGF modulates the PDGF-driven pathway of oligodendrocyte development. Neuron 5:603-614.

Menn B, Garcia-Verdugo JM, Yaschine C, Gonzalez-Perez O, Rowitch D, Alvarez-Buylla A (2006) Origin of oligodendrocytes in the subventricular zone of the adult brain. J Neurosci 26:7907-7918.
Merkle FT, Mirzadeh Z, Alvarez-Buylla A (2007) Mosaic organization of neural stem cells in the adult brain. Science 317:381-384.

Mirzadeh Z, Merkle FT, Soriano-Navarro M, Garcia-Verdugo JM, AlvarezBuylla A (2008) Neural stem cells confer unique pinwheel architecture to the ventricular surface in neurogenic regions of the adult brain. Cell Stem Cell 3:265-278.

Nishiyama A, Lin XH, Giese N, Heldin CH, Stallcup WB (1996) Colocalization of NG2 proteoglycan and PDGF alpha-receptor on O2A progenitor cells in the developing rat brain. J Neurosci Res 43:299-314.

Nistér M, Heldin CH, Westermark B (1986) Clonal variation in the production of a platelet-derived growth factor-like protein and expression of corresponding receptors in a human malignant glioma. Cancer Res 46:332-340.

Palmer TD, Takahashi J, Gage FH (1997) The adult rat hippocampus contains primordial neural stem cells. Mol Cell Neurosci 8:389-404.

Parras CM, Hunt C, Sugimori M, Nakafuku M, Rowitch D, Guillemot F (2007) The proneural gene Mash1 specifies an early population of telencephalic oligodendrocytes. J Neurosci 27:4233-4242.

Pastrana E, Cheng LC, Doetsch F (2009) Simultaneous prospective purification of adult subventricular zone neural stem cells and their progeny. Proc Natl Acad Sci U S A 106:6387-6392.

Pincus DW, Harrison-Restelli C, Barry J, Goodman RR, Fraser RA, Nedergaard M, Goldman SA (1997) In vitro neurogenesis by adult human epileptic temporal neocortex. Clin Neurosurg 44:17-25.

Pringle NP, Richardson WD (1993) A singularity of PDGF alpha-receptor expression in the dorsoventral axis of the neural tube may define the origin of the oligodendrocyte lineage. Development 117:525-533.

Rakic S, Zecevic N (2003) Early oligodendrocyte progenitor cells in the human fetal telencephalon. Glia 41:117-127.

Reynolds BA, Weiss S (1992) Generation of neurons and astrocytes from isolated cells of the adult mammalian central nervous system. Science 255:1707-1710.

Sanai N, Tramontin AD, Quiñones-Hinojosa A, Barbaro NM, Gupta N, Kunwar S, Lawton MT, McDermott MW, Parsa AT, Manuel-García Verdugo J, Berger MS, Alvarez-Buylla A (2004) Unique astrocyte ribbon in adult human brain contains neural stem cells but lacks chain migration. Nature 427:740-744.

Shen Q, Wang Y, Kokovay E, Lin G, Chuang SM, Goderie SK, Roysam B, Temple S (2008) Adult SVZ stem cells lie in a vascular niche: a quantitative analysis of niche cell-cell interactions. Cell Stem Cell 3:289-300.

Wolswijk G, Noble M (1989) Identification of an adult-specific glial progenitor cell. Development 105:387-400. 